\title{
Incidence of genes encoding vanA/vanB vancomycin resistance in rectal swabs of patients with diagnosed cancer, on the day of admission to hospital, in a non-epidemic period
}

\author{
Maria Szymankiewicz ${ }^{1}$, Joanna Wróblewska ${ }^{1}$, Tomasz Nowikiewicz ${ }^{2,3}$ \\ ${ }^{1}$ Department of Microbiology, Prof. F. Łukaszczyk Centre of Oncology, Bydgoszcz, Poland \\ ${ }^{2}$ Chair and Clinic of Oncological Surgery, Collegium Medicum in Bydgoszcz, Nicolaus Copernicus University in Torun, Poland \\ ${ }^{3}$ Clinical Department of Breast Cancer and Reconstructive Surgery, Prof. F. Łukaszczyk Centre of Oncology, Bydgoszcz, Poland
}

Key words: colonisation, vanA/vanB enterococci, oncological patients.

Address for correspondence: Maria Szymankiewicz PhD, Department of Microbiology, Prof. F. Łukaszczyk Centre of Oncology, Bydgoszcz, Poland, e-mail: szymankiewiczm@co.bydgoszcz.pl

\begin{abstract}
Introduction: Rapid diagnosis is important for preventing infections due to vancomycin-resistant enterococci.

Aim: To evaluate the status of gastrointestinal colonisation with strains containing vanA/vanB genes in oncological patients. Material and methods: A total of 167 samples of rectal swabs taken from 161 patients (mean age: 63, range: 29-93 years) were examined, including 113 patients from surgical wards (70.2\%) and 48 patients from non-surgical wards (29.8\%), with diagnosed cancer. The tests were carried out within $24 \mathrm{~h}$ of admitting the patient to the department, using the Cepheid Xpert vanA/vanB test, with a CE marked GeneXpert ${ }^{\oplus}$ Instrument Systems analyser. Samples with positive vanB gene results were additionally seeded on chromogenic media.

Results: The presence of the vanA gene was found in $2.7 \%$ and $6.3 \%$ of the examined patients, respectively, from the surgical and non-surgical departments, which accounted for $3.7 \%$ of all the patients examined. The presence of the vanB gene was detected in $21.1 \%$ of the patients, but in no case was there any growth of vancomycin-resistant enterococci on the chromogenic medium.

Conclusions: Patients admitted to non-surgical wards were more often colonised with vanA/vanB genes than were patients admitted to surgical wards, but the differences were not statistically significant.
\end{abstract}

\section{Introduction}

Bacteria of the genus Enterococcus, especially Enterococcus faecalis (E. faecalis) and Enterococcus faecium (E. faecium), are an important aetiological factor of nosocomial infections, including bacteraemia, urinary tract infections, and intra-abdominal and pelvic infections [1, 2]. According to data reported in the EARS-Net (European Antimicrobial Resistance Surveillance Network) for Poland for the year 2017, vancomycin-resistant strains (vancomycin-resistant Enterococcus - VRE) account for $31.5 \%$ of all $E$. faecium isolates and $3.0 \%$ of $E$ faecalis isolates originating from invasive infections. The increase in the percentage of VRE among enterococci in the period 2014-2017 given in this report is highly dis- turbing [3]. The evolving epidemiology of enterococcal infections and the observed increase in the proportion of vancomycin-resistant $E$. faecium present new challenges for hospital infection control teams aiming to reduce the risk of spreading vancomycin-resistant enterococci.

One of the ways to reduce the transmission of VRE within a hospital is to screen for it when patients are admitted to the hospital. However, these tests are not recommended for all admitted patients. The legitimacy and expediency of performing the tests should be considered with regard to the local epidemiological situation and the existence of risk factors for colonisation of VRE [4]. It is known, however, that oncological patients are a high-risk group for VRE colonisation. This is due 
to the frequent use of antibiotics, comprehensive (often extensive) surgery, combined treatment (surgery, chemotherapy, radiotherapy), immunosuppression, neutropenia, and long hospitalisations [5].

Rapid identification of VRE-colonised patients using tests detecting vancomycin-resistance genes accelerates the implementation of appropriate procedures in the area of hospital infection control, including contact isolation, due to the short waiting time for results, which may limit the spread of microorganisms between patients $[6,7]$.

\section{Aim}

The aim of our work was to analyse the status of colonisation of enterococci that have vancomycin-resistant vanA/vanB genes in gastrointestinal tract patients admitted to one oncological centre within a 12-month period. In addition, we assessed whether there were statistically significant differences in the occurrence of vanA/van $B$ genes in patients admitted to the surgical and non-surgical departments.

\section{Material and methods}

A total of 167 samples of rectal swabs taken from 161 patients were examined, including 113 (70.2\%) patients from surgical wards (oncological surgery, oncological gynaecology, chest surgery, and lung cancer) and $48(29.8 \%)$ patients from non-surgical wards (chemotherapy, oncology, radiotherapy) in the period from April 2018 to March 2019, within 24 h of admission to the ward, in one oncological centre. All patients with diagnosed cancer were admitted to the hospital for surgical and/or complementary treatment (cytotoxic, cytostatic, radiotherapy).

An epidemiological interview was conducted; patients who met certain criteria on admission to the hospital were qualified for screening. Each patient filled in a questionnaire in the admission room, which was verified by the nurse admitting the patient to the ward. Rectal swabs were collected if at least one of the following criteria was met: hospitalisation in the last year outside of the centre; residence in the last year in longterm care facilities; and exposure to two or more antibiotics over the last 30 days. Studies were performed only in patients with recognised risk factors for VRE carriers. The swabs were immediately transferred to the Microbiology Department of the centre, where the Cepheid Xpert vanA/vanB test was carried out with the CE marked GeneXpert ${ }^{\oplus}$ Instrument Systems analyser. The Cepheid Xpert vanA/vanB real-time PCR test detects genes related to vancomycin $\operatorname{van} A / \operatorname{van} B$ resistance and provides results in less than $1 \mathrm{~h}$. In the event of an erroneous result (Error) the test was repeated within
$2 \mathrm{~h}$. In accordance with the procedure followed in the centre, when vanB was positive, due to the low specificity of the test and low positive predictive value, the culture method was used as a confirmatory test. Such proceedings were in accordance with the recommendations of the manufacturer. The sample was taken within $2 \mathrm{~h}$ of a positive Cepheid test. Specimens were plated onto chromogenic substrate Brilliance ${ }^{\text {TM }}$ VRE Agar (Oxoid, US), on which the growth of Enterococcus faecalis and Enterococcus faecium was evaluated after $24 \mathrm{~h}$ and $48 \mathrm{~h}$ incubation. A negative result was reported after $48 \mathrm{~h}$. Until the result was obtained, the patients were isolated, and if a positive result was obtained, contact precautions were implemented in order to minimise contact with VRE-positive patients. Patients with a positive result for VRE were reported to an epidemiological nurse. No VRE epidemic was reported during the study period.

\section{Statistical analysis}

Statistical analysis of the relationship between the type of ward (surgical vs. non-surgical) and the occurrence of positive results vanA/vanB was carried out using Fisher's exact test. Differences were accepted as statistically significant when the significance level was less than $0.05(p<0.05)$. The results are also shown as percentages.

The study received approval from the Bioethical Commission at the Collegium Medicum of Nicolaus Copernicus University in Torun (KB approval number 401/2019).

\section{Results}

For six samples (3.7\%) from six patients, amplification of the target vanA sequences reached the $\mathrm{Ct}$ limit cycle in the normal range, and the final fluorescence reading was above the threshold value: a positive result was obtained. The target DNA vanB was detected in 34 (21.1\%) samples from 34 patients, with a negative vanA result, and with one positive result obtained after repeated testing. Thirty of the 34 samples in which the van $B$ gene was detected were seeded on chromogenic media without obtaining confirmation of the presence of vancomycin-resistant enterococci. In 4 cases in which vanB DNA was detected, no samples were obtained for retesting. The reason was the patient's refusal to give another rectal swab sample.

For 6 (3.7\%) samples, there was an Error - syringe pressure reading exceeded protocol limit, which was probably the result of a pressure sensor error or clogging of the filter due to debris in the sample. Following the manufacturer's instructions, the tests were repeated using a new cartridge, after previous swab dilution. After 
Table I. Xpert vanA/vanB test - results in relation to the ward type

\begin{tabular}{lccc} 
Ward type & $\begin{array}{c}\text { No. of samples } \\
\text { tested }\end{array}$ & $\begin{array}{c}\operatorname{vanA}(+) \\
n(\%)\end{array}$ & $\begin{array}{c}\operatorname{vanB}(+) \\
n(\%)\end{array}$ \\
\hline Non-surgical ward & 48 & $3(6.3)$ & $13(27.1)$ \\
\hline Surgical ward & 113 & $3(2.7)$ & $21(18.6)$ \\
\hline Total & 161 & $6(3.7)$ & $34(21.1)$
\end{tabular}

repeating the tests, in 1 case $(0.6 \%$ of all samples tested), an error was again obtained, while in the remaining 5 cases there were four negative results and one positive result for the presence of the van $B$ gene (not confirmed by positive enterococci culture with the $\operatorname{van} B$ phenotype). There were no statistically significant differences in the frequency of VRE vanA or vanB colonisation between the surgical and non-surgical departments $(p>0.05)$. The occurrence of VRE vanA/vanB colonisation depending on the ward type is shown in Table I.

\section{Discussion}

In our work, the presence of the vanA gene was found in $3.7 \%$ of all patients examined. The presence of the $v a n B$ gene was detected in $1 / 5$ of the patients enrolled in the study, but no growth of vancomycin-resistant enterococci on the chromogenic medium was found. Our results also confirm the observation of other authors that the Cepheid Xpert vanA/vanB test has a low positive predictive value (PPV) for van $B$ enterococci.

The frequency of VRE colonisation among patients admitted to hospital varies from $3.6 \%$ to $30.0 \%$ and depends on the profile of the hospital, ward, period in which the tests are performed (epidemic vs. non-epidemic), as well as risk factors associated with the disease and treatment [8-12]. Factors that increase the risk of gastrointestinal VRE colonisation include previous treatment with antibiotics, mainly carbapenems, fluoroquinolones, third-generation cephalosporins (ceftriaxone, ceftazidime), and glycopeptides (vancomycin) and platinum analogues; previous hospitalisations, especially in the intensive care unit; and the patient's age [10, 13]. Risk factors are also surgery and immunosuppression [14]. However, in our work we did not attempt to analyse the risk factors facilitating VRE colonisation. The detailed risk factors collected did not allow for their reliable analysis, and therefore were not included in this work.

The frequency of colonisation varies widely. The highest percentage of VRE colonisation is found in patients from intensive care units, up to $30.0 \%$, and the lowest in patients from oncological haematology units, about $5.0 \%[11,12,15]$. Continuous admission of
VRE colonised patients to wards is crucial for endemic persistence of VRE within the ward. Therefore, in our study an attempt was made to assess which wards of the oncology hospital are more likely to admit patients colonised with VRE. The higher percentage of VRE colonised patients found among those admitted to non-surgical departments than to surgical departments is probably connected with the patients' characteristics, most of whom were admitted to continue treatment with chemotherapy or radiotherapy.

In patients undergoing treatment with cytostatics, the mucosal barrier of the gastrointestinal tract is disturbed, resulting in microbial translocation and the development of systemic infection. A relationship between colonisation and subsequent infection was found in patients of haematological-oncology wards, patients with leukaemia, and patients undergoing stem cell transplantation. This phenomenon has been documented in the case of blood and urinary tract infections, and infections were significantly more frequent in patients previously VRE colonised than in those who were non-colonised. The development of infection is also favoured by episodes of febrile neutropaenia, which require the application of antibiotic therapy, including the use of vancomycin $[11,12]$. In our study, we did not address later complications in the form of infections, but nevertheless it seems that a higher percentage of VRE colonisation in the group of non-surgical patients, often after previous surgery, antibiotic therapy, and before complementary anticancer treatment, may be a potential risk of developing infection. However, further investigation is necessary to confirm this thesis.

The presence of vanB was high in our patients: over $1 / 4$ and about $1 / 5$ patients in non-surgical and surgical wards, respectively, had positive results. However, this was not confirmed by growth on chromogenic agar. This result is in accordance with previous studies, and possible explanations for this result are limitation of the Cepheid test or the presence of uncultured bacteria or anaerobic bacteria in the specimen $[9,15,16]$.

In active infection control programs, to assess VRE colonisation, there is a preference for molecular biology methods and real-time PCR, which give a result in up to $1 \mathrm{~h}$ and the possibility to quickly implement contact isolation. Tests, however, should be characterised by high sensitivity and acceptable specificity. The sensitivity and specificity of the Cepheid Xpert vanA/vanB test as well as the positive (PPV) and negative (NPV) predictive values were the subject of several works. Holzknecht et al. [9] evaluated the sensitivity and specificity of the vanA test at 87.1 and $99.7 \%$ for PPV and NPV, respectively, at 98.0 and $97.7 \%$, while in the case of the $\operatorname{van} B$ test the authors assessed the specificity and PPV at the level of 
77.6 and $0.4 \%$ respectively, performing tests on over 1000 samples from patients from four Danish university hospitals. On the other hand, in Gazin et al. [16], involving a smaller number of test samples from patients undergoing dialysis, the sensitivity and specificity of the vanA test was determined at 73.9 and $92.6 \%$ and for the $\operatorname{van} B$ test, 87.5 and $14.7 \%$, respectively. The authors, however, emphasise that the test shows high sensitivity when detecting VRE at low concentrations of 10-100 colony forming units (CFU/ml).

The false positive results of the $\operatorname{van} B$ test are related to the lack of specificity of the primers used in the test [15] and, above all, the presence of other microorganisms in the test material, having genes with a high level of similarity or identical to Enterococcus vanB $[17,18]$. In our study, positive results for vanB were in no case confirmed in VRE culture on chromogenic medium - we found no true positive results. Our observations confirm the results of Żabicka et al. [19], who recommend that in the case of a positive result for $\operatorname{van} B$, the status of the carrier should be confirmed using the culture method, which is particularly important during an epidemic period.

\section{Conclusions}

This study provides information about vanA/vanB colonisation in the gastrointestinal tract among selected oncological patients admitted within one year to a single oncological hospital. Patients admitted to non-surgical wards were more often colonised with vanA/vanB strains than patients of surgical wards; however, the differences were not statistically significant. The presence of $\operatorname{van} B$, however, was not confirmed by the culture method, which limits the use of the test for quick identification of vanB Enterococcus and makes it impossible to make a clear decision about the need to isolate the patient. This study has two limitations: one is the difference in the number of patients between groups, and the other is the lack of detailed analysis of risk factors for VRE colonisation.

\section{Conflict of interest}

The authors declare no conflict of interest.

\section{References}

1. O'Driscoll T, Crank CW. Vancomycin-resistant enterococcal infections: epidemiology, clinical manifestations, and optimal management. Infect Drug Resist 2015; 8: 217-30.

2. Matar MJ, Tarrand J, Raad I, et al. Colonization and infection with vancomycin-resistant enterococcus among patients with cancer. Am J Infect Control 2006; 34: 534-6.

3. Surveillance of antimicrobial resistance in Europe. Annual report of the European Antimicrobial Resistance Surveillance Network (EARS-Net) 2017. https://ecdc.europa.eu/en/publi- cations-data/surveillance-antimicrobial-resistance-europe2017.pdf (November 2018).

4. Zalecenia prowadzenia mikrobiologicznych badań przesiewowych u hospitalizowanych pacjentów, Narodowy Program Ochrony Antybiotyków na lata 2016-2020, NIL, Warszawa 2017, https://antybiotyki.edu.pl/wp-content/uploads/ Rekomendacje/drZALECENIAPROWADZENIABADAN13.07.pdf.

5. Ariza-Heredia EJ, Chemaly RF. Infection control practices in patients with hematological malignancies and multidrug-resistant organisms: special considerations and challenges. Cl Lymph Myelom Leuk 2014; 14 (Suppl): 104-10.

6. Kampmeier S, Knaack D, Kossow A, et al. Weekly screening supports terminating nosocomial transmissions of vancomycin resistant enterococci on an oncologic ward - a retrospective analysis. Antimicrob Resist Infect Control 2017; 6: 48.

7. Werner G, Coque TM, Hammerum AM, et al. Emergence and spread of vancomycin resistance among enterococci in Europe. Euro Surveill 2008; 13: pii: 19046.

8. Uludağ Altun H, Ataman Hatipoğlu Ç, Bulut C, et al. Surveillance of vancomycin-resistant Enterococci colonization with GeneXpert vanA/vanB test and culture method. J Microbiol Infect Dis 2014; 4: 97-101.

9. Holzknecht BJ, Hansen DS, Nielsen L, et al. Screening for vancomycin - infection control decision making. $N$ Microbes N Infect 2017; 16: 54-9.

10. Liss BJ, Vehreschild JJ, Cornely OA, et al. Intestinal colonisation and blood stream infections due to vancomycin-resistant enterococci (VRE) and extended spectrum beta-lactamase-producing Enterobacteriaceae (ESBLE) in patients with haematological and oncological malignancies. Infection 2012; 40: 613-9.

11. Rolston KV, Nesher L, Mulanovich V, et al. Intestinal colonisation and blood stream infections due to vancomycin-resistant enterococci (VRE) and extended-spectrum beta-lactamase-producing enterobacteriaceae (ESBLE) in patients with haematological and oncological malignancies. Infection 2013; 41: 1039-40.

12. Amberpet R, Sistla S, Parija CS, et al. Screening for intestinal colonization with vancomycin eesistant enterococci and associated risk factors among patients admitted to an Adult Intensive Care Unit of a Large Teaching Hospital. J Clin Diagn Res 2016; 10: DC06-9.

13. Flokas ME, Karageorgos SA, Detsis M, et al. Vancomycin-resistant enterococci colonization, risk factors and risk for infection among hospitalized paediatric patients: a systematic review and meta-analysis. Int J Antimicrob Agents 2017; 49: 565-72.

14. Amberpet R, Sistla S, Parija CS, et al. Risk factors for intestinal colonization with vancomycin resistant enterococci' A prospective study in a level III pediatric intensive care unit. J Labor Phys 2018; 10: 89-94.

15. Yağci S, Ataman Hatipoğlu C, Altun S, et al. Evaluation of Gene Xpert vanA/vanB assay for the detection of vancomycin-resistant enterococci in patients newly admitted to intensive care units. Turk J Med Sci 2013; 43: 1008-12.

16. Gazin M, Lammens C, Goossens H, et al.; MOSAR WP2 Study Team (2012). Evaluation of GeneOhm VanR and Xpert vanA/ vanB molecular assays the rapid detection of vancomycin-resistant enterococci. Eur J Clin Microbiolol Infect Dis 2012; 31: 273-6. 
17. Stinear TP, Olden DC, Johnson PD, et al. Enterococcal vanB resistance locus in anaerobic bacteria in human faeces. Lancet 2001; 357: 855-6.

18. Morris KA, Macfarlane-Smith LR, Wilcox MH. Evaluation of the novel artus C. difficile QS-RGQ, VanR QS-RGQ and MRSA/SA QS-RGQ assays for the laboratory diagnosis of Clostridium difficile infection (CDI), and for vancomycin-resistant enterococci (VRE) and methicillin-resistant Staphylococcus aureus (MRSA) screening. Eur J Clin Microbiol Infect Dis 2017; 36: 823-9.

19. Żabicka D, Strzelecki J, Woźniak A, et al. Efficiency of the Cepheid Xpert vanA/vanB assay for screening of colonization with vancomycin-resistant enterococci during hospital outbreak. Antonie van Leeuwenhoek 2012; 101: 671-5.

Received: 15.07 .2019

Accepted: 20.09.2019 